\title{
Peroral endoscopic myotomy: a literature review and the first UK case series
}

\author{
Authors: Shraddha Gulati, ${ }^{A}$ Andrew Emmanuel, ${ }^{A}$ Haruhiro Inoue,${ }^{B}$ Bu'Hussain Hayee ${ }^{C}$ and Amyn Haji ${ }^{D}$
}

\begin{abstract}
Peroral endoscopic myotomy (POEM) is an established treatment for primary achalasia. It has gained endorsement from the American Society for Gastrointestinal Endoscopy with increasing clinical acceptance since the first procedure, performed in Japan in 2008. The first successful POEM in the UK was performed in November 2013 at King's College Hospital and this article presents the first UK case series. Prospective data were collected at 3 and 12-24 months for consecutive patients undergoing POEM. Post-POEM gastro-oesophageal reflux health-related quality of life scale (GORD-HRQoL) score was recorded. Statistical comparisons were made using paired non-parametric testing. In an initial series of 33 consecutive prospectively followed patients (12 female; $49.5 \pm 13$ years; median follow-up 9 (3-28) months; $58 \%$ having had previous intervention), a $91 \%$ success rate has been achieved at 3 months. To date, 16 patients have reached the 12 -month time point, with $13(81 \%)$ sustaining response. This case series compares well with international cohorts and demonstrates excellent long-term safety and favourable efficacy.
\end{abstract}

KEYWORDS: Achalasia, endoscopy, manometry, oesophageal motility disorders

\section{Introduction}

Primary achalasia occurs secondary to loss of the postganglionic inhibitory neurons of the myenteric plexus. This impairs lower oesophageal sphincter (LOS) relaxation in response to the ingestion of solids and liquids. It has an incidence of 1 in 100,000 adults worldwide with an equal gender distribution. ${ }^{1}$ The clinical features - which can be misinterpreted and treated as reflux, leading to a delay in recognition and treatment include dysphagia to both solids and liquids, retrosternal pain, regurgitation and weight loss. Clinical severity and response to treatment is measured using the Eckardt score. ${ }^{2}$

A series of investigations are required to confirm the diagnosis and exclude main differentials of pseudoachalasia and reflux.

Authors: ${ }^{\text {A }}$ linical research fellow, King's Institute of Therapeutic Endoscopy, London, UK; ${ }^{\mathrm{B}}$ director, Digestive Diseases Centre, Showa University Koto-Toyosu Hospital, Tokyo, Japan; ${ }^{C}$ consultant gastroenterologist and advanced endoscopist, King's Institute of

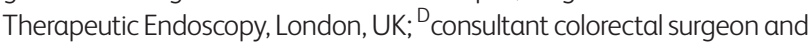
endoscopy lead, King's Institute of Therapeutic Endoscopy, London, UK
These include contrast swallow radiography illustrating the classical 'birds-beak' appearance (Fig 1A) and gastroscopy, which may demonstrate a dilated oesophagus with the impression of a tight LOS. The gold standard diagnostic test is high resolution manometry, which allows subcategorisation using the Chicago classification. Type I 'classical' achalasia is characterised by incomplete lower oesophageal relaxation and absent peristalsis, type II exhibits pan-oesophageal pressurisation and is most prevalent (Fig 1B), while type III exhibits evidence of oesophageal spasm with premature oesophageal contractions. ${ }^{3}$

Medical therapy for achalasia with nitrate-donors and calcium channel blockers is ineffective. 'Simple' endoscopic therapy involves pneumatic dilatation (PD; causing mechanical disruption of the muscular fibres of the LOS) and injection of botulinum toxin (BTX) at the LOS, inhibiting excitatory neurotransmitter release at the presynaptic membrane. ${ }^{4}$ The effect of BTX is short-lived in comparison with PD; however, PD may necessitate repetition with additional risk of oesophageal perforation. ${ }^{5}$ In patients fit for surgery, laparoscopic Heller myotomy (LHM) has been regarded as the definitive treatment. ${ }^{6,7}$ The long-term success of $\mathrm{PD}$ is comparable to surgery if the need for repeat procedures is acknowledged (ie not regarded as a treatment failure) and the former is applied using a standardised protocol. ${ }^{8}$ Post-procedure gastro-oesophageal reflux disease (GORD) is a reasonable concern in any therapy that mechanically disrupts the LOS and LHM is commonly combined with fundoplication to address this. ${ }^{9}$

\section{Peroral endoscopic myotomy for achalasia}

Ortega et $a l^{10}$ first reported an endoscopic approach to myotomy in 1980. In 2007, Pasricha et al ${ }^{11}$ published a porcine model for endoscopic myotomy followed by the first POEM performed on a patient with achalasia in Japan in 2008 by Professor Haru Inoue. ${ }^{12}$ POEM offers an external incisionless procedure, achieving a myotomy of greater length than that possible by a laparoscopic surgical approach and has been endorsed by the American Society of Gastrointestinal Endoscopy as a safe and efficacious treatment option. ${ }^{13}$ There is no current age limit for the procedure: the youngest patient to date being 3 years and the oldest 93 years old. ${ }^{14,15}$ Of particular note is the versatility and efficacy in patients who have undergone previous endoscopic therapy or LHM, and indications for the procedure have expanded to include a spectrum of spastic oesophageal motility disorders. ${ }^{16-18}$ Contraindications to POEM include refractory 


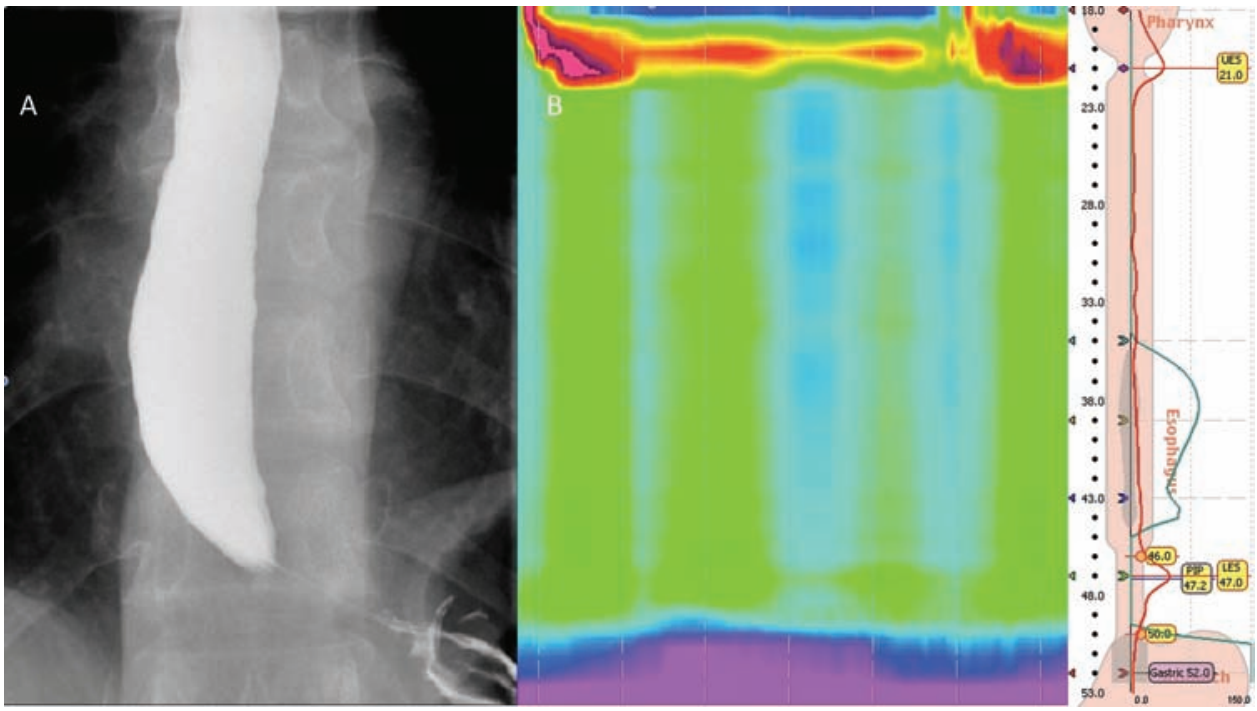

Fig 1. A - Barium swallow radiograph illustrating classical 'bird beak' appearance in achalasia; B - high resolution manometry illustrating the most common subtype, type II achalasia.

achalasia with mega-oesophagus, significant oesophageal scarring secondary to oesophageal radiation therapy, oesophageal mucosal resection or ablation. Comorbidities contraindicating general anaesthesia must also be considered: severe pulmonary disease, coagulopathy and cirrhosis with portal hypertension for instance. ${ }^{19}$

The first POEM successfully performed in the UK was at King's College Hospital, London in November 2013. This article presents a prospective analysis of the first 33 cases.

\section{Methods}

\section{Patient selection}

Patients were investigated for symptoms suggestive of achalasia with a barium swallow and gastroscopy. High resolution manometry confirmed and subcategorised the diagnosis of achalasia. Patients were counselled for POEM in a dedicated clinic where all treatment options were discussed, taking into account patient preference, comorbid conditions and contraindications. All patients gave consent to undergo POEM. Prospective data were collected for consecutive patients preand 3 months post-procedure in all cases. Additionally, longterm follow up of 12-24 months post-POEM were recorded.

\section{Patient preparation}

All patients observed a liquid diet for 48 hours and were subsequently nil by mouth 6 hours prior to the procedure. All procedures were performed in the main operating theatre under general anaesthetic.

Approval from the local research ethics committee was not required as there was no deviation from standard patient care. Informed consent was not required as this is an anonymised case series presentation.

\section{POEM technique}

The procedure undertaken was identical to that previously described(Fig 2). ${ }^{12}$ Confirmation of completeness of myotomy
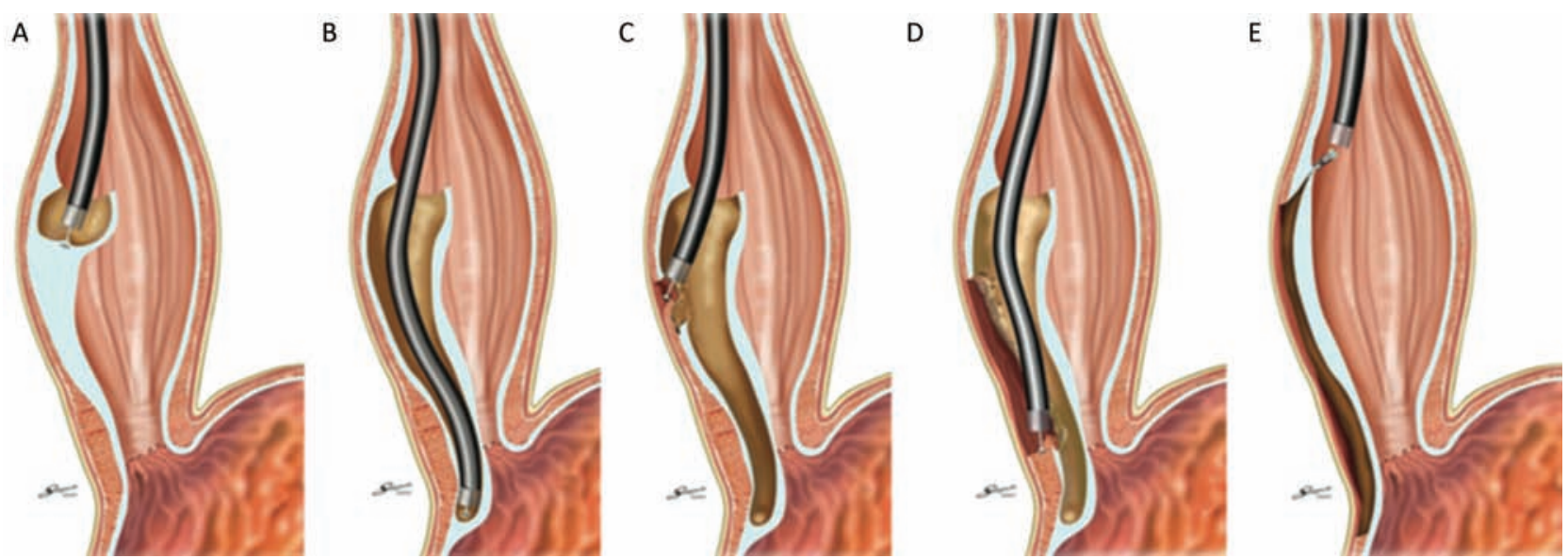

Fig 2. Schematic diagram of POEM procedure. A - mucosal entry; B - creation of submucosal tunnel; C,D - myotomy; E - closure of mucosal entry. Reproduced with permission from Inoue et al. ${ }^{13}$ 

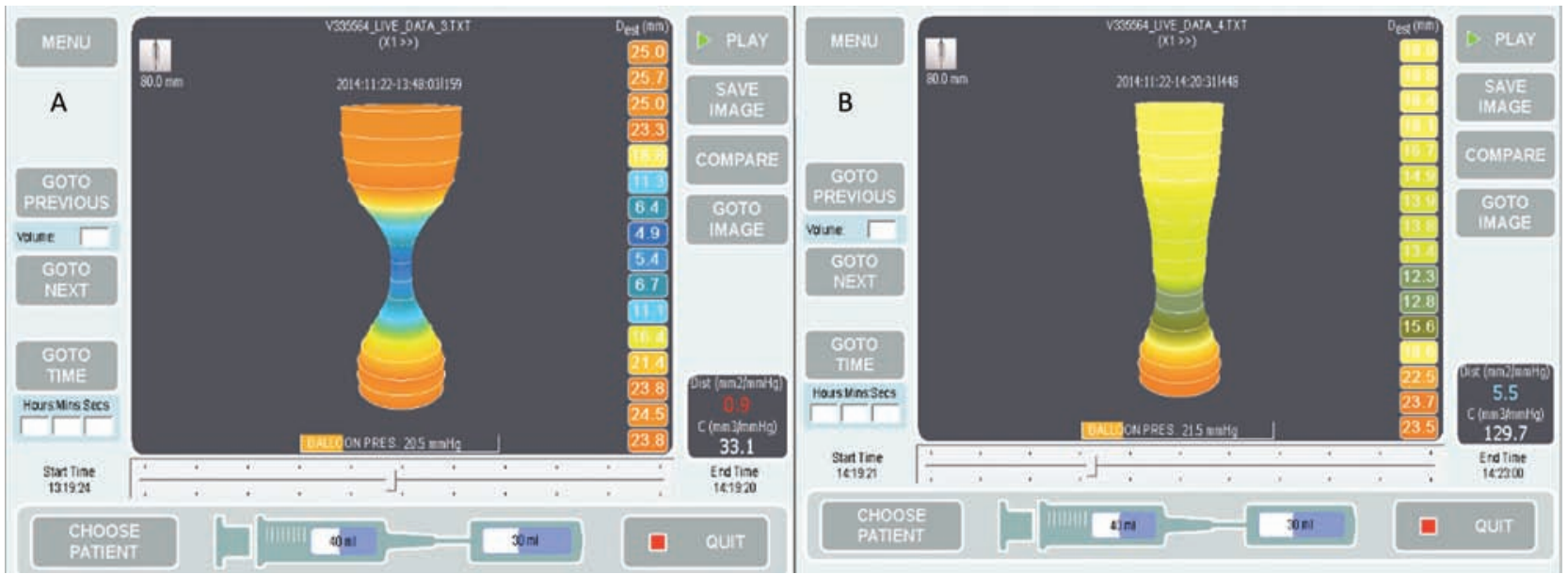

Fig 3. EndoFLIP® pre- and post-myotomy. A - Pre-myotomy gastro-oesophageal junction diameter $5.4 \mathrm{~mm}$; B - post-myotomy gastro-oesophageal junction diameter $12.8 \mathrm{~mm}$.

is a key step and although the myotomy is usually extended $2-3 \mathrm{~cm}$ beyond the LOS into the gastric submucosa, it can be challenging to establish this relying on anatomical landmarks alone. Completeness is most reliably achieved by the 'double scope technique' where the submucosal tunnel is intubated with an ultra-slim gastroscope and the oesophagus intubated concomitantly with an adult gastroscope and retroflexed in the fundus. Using transillumination of the paediatric gastroscope in the submucosal tunnel, the extent of the submucosal tunnel on the gastric side of the LOS is confirmed. ${ }^{20}$ This was used in four challenging cases because of previous treatment and one with significant fibrosis.

Another technique employs the endoluminal functional lumen imaging probe (EndoFLIP ${ }^{\circledR}$, Crospon Technologies, Galway, Ireland). The balloon probe is inserted through the oesophageal lumen to evaluate the gastro-oesophageal junction distensibility using impedance planimetry in response to volume distension. This is used pre- and post-myotomy and provides a real-time opportunity to assess the effectiveness of myotomy. ${ }^{21}$ To date, this has been performed successfully in nine patients in our series (Fig 3). The clinical utility of this has yet to be established although a real-time assessment of disruption of the LOS is an attractive adjunct to the endoscopic method.

\section{Post-procedure}

All patients were kept nil-by-mouth until a contrast swallow and upper gastrointestinal endoscopy were obtained on postprocedure day 1 . These procedures exclude mucosal breaches (contrast leak), laryngeal inflammation, early reflux changes or mucosal injury. All endoscopic clips used to close the mucosal entry site were accounted for and replaced if necessary.

Once early complications were excluded, all patients commenced oral free fluids for the first week postoperatively, followed by a soft/purée diet for the second week, resuming a normal diet thereafter.

Clinical success was defined using the accepted criteria of a reduction of Eckardt score (ES) to $\leq 2$ or a reduction in 4 points from baseline, at planned post-procedure assessments
(3 and 12-24 months). ${ }^{16}$ Secondary considerations included documenting a reduction in both 4-second integrated lower oesophageal relaxation pressure (4sIRP) and ES between 12-24 months post-POEM.

Post-POEM gastro-oesophageal reflux was screened for using the gastro-oesophageal reflux health-related quality of life scale (GORD-HRQoL) and was repeated to measure response to therapy. ${ }^{22}$

Means and standard deviations were used to describe continuous variables with medians and ranges used for categorical variables. The Wilcoxon signed ranks test was used to compare pre- and post-procedure differences in ES and 4 sIRP and to compare pre- and post-treatment differences in GORD-HRQoL score. Repeated-measures ANOVA was used for multiple time-point comparisons.

\section{Results}

Prospective data from 33 patients were analysed. Patient demographics and baseline characteristics are presented in Table 1.

Median gastric, oesophageal and total myotomy length were $3 \mathrm{~cm}$ (range 2-4), $10 \mathrm{~cm}$ (range 5-16) and $13 \mathrm{~cm}$ (range 8-19), respectively. 'Anterior' myotomy was performed in 28/33 $(85 \%)$ with the posterior approach chosen in the remaining patients (who had undergone previous myotomy). Selective circular myotomy was performed in all cases.

To date, follow-up has extended beyond the 3-month and 12-24 month assessments for 32 and 16 patients, respectively (median follow up 9 (range 3-28) months). One patient required revision of POEM at 6 months because of persistent symptoms; ES 11 (dysphagia 3, chest pain 3, weight loss 3, regurgitation 2) but has now achieved symptomatic relief (ES 2 post-procedure).

\section{Eckardt scores}

Median pre- and post-POEM ( 3 months) ES were 8 (range $3-12$ ) and 0 (range $0-6, p<0.0001$, Fig 4 A). POEM was clinically successful in 29/32 (91\%) patients at 3 months. 


\section{Table 1. Baseline characteristics}

\begin{tabular}{ll} 
Patient demographics & \\
Mean age (range), years & $49.5 \pm 13(22-79)$ \\
Male & $21(64)$ \\
Female & $12(36)$ \\
Clinical data & \\
Mean duration of disease (range), years & $4.9 \pm 5.68(0.25-25)$ \\
Median Eckardt Score (range) & $8\left(3^{*}-12\right)$ \\
Chicago subcategorisation & \\
Achalasia type I & $2(6)$ \\
Achalasia type II & $31(94)$ \\
Non-sigmoid oesophagus & $28(85)$ \\
Sigmoid oesophagus & $5(5)$ \\
Treatment history & \\
Prior achalasia treatment & $19(58)$ \\
Prior botulinum toxin injection & $8(24)$ \\
Prior pneumatic dilatation & $10(30)$ \\
Prior Heller myotomy & $4(12)$ \\
Prior POEM & $1(3)$ \\
$>2$ prior treatments & $2(6)$ \\
ASA physical status classification & \\
ASA grade I & $8(24)$ \\
ASA grade II & $19(58)$ \\
ASA grade III & $6(18)$ \\
\hline Aldan (\%) unss stan & \\
\hline
\end{tabular}

All data $n(\%)$ unless stated otherwise.

*shortly post-pneumatic dilatation required repeatedly. In between pneumatic dilatation, Eckardt Score was 12.

$\mathrm{ASA}=$ American Society of Anesthesiologists; $\mathrm{POEM}=$ peroral endoscopic myotomy

Seventeen patients were eligible for analysis between 12 and 24 months post-POEM. Data for one of seventeen 12-24 month patients was not available because of loss to follow-up. Sustained response has been observed in the remaining 16 patients
(Fig 4B): ES 8 (range 5-12) versus 2 (range $0-7, \mathrm{p}=0.001$ ), ANOVA $\mathrm{p}<0.0001$. Therefore, sustained clinical success has been confirmed in $13 / 16$ cases to date ( $81 \%)$.

Patient demographics, clinical and procedural data of patients in whom clinical success was not achieved at the 3 month review $(\mathrm{n}=3)$ or sustained at the $12-24$ month review $(\mathrm{n}=3)$ are presented in Table 2.

\section{Manometric data}

A significant reduction in 4sIRP has been documented:

$25.3 \pm 11.01 \mathrm{mmHg}$ versus $9.267 \pm 5.489 \mathrm{mmHg}(\mathrm{p}=0.004$, Fig 4C).

\section{EndoFlip}

Pre and post-myotomy gastro-oesophageal junction diameter increased from $5.8 \mathrm{~mm}$ to $12.12 \mathrm{~mm}(\mathrm{n}=5, \mathrm{p}=0.0625)$ and distensibility improved from $1.37 \mathrm{~mm}^{2} / \mathrm{mmHg}$ to $2.56 \mathrm{~mm}^{2}$ / $\operatorname{mmHg}(\mathrm{n}=3, \mathrm{p}=0.25)$.

\section{Adverse events}

One patient experienced displacement of a single clip, identified and replaced at the check endoscopy (day 1 post-POEM) without further sequelae. There were no cases of mortality, perforation, pneumomediastinum or mucosal injury. Median length of hospital stay was 3 nights (range 1-12) with no hospital re-admissions within 30 days.

\section{Gastro-oesophageal reflux disease}

Median postoperative GORD-HRQoL score $(n=29)$ was 3 (range 0-31). Eight patients scored above 12 in the GORDHRQoL scale. Significant improvement was seen by comanagement with proton pump inhibitor and dietary modification: $15.5(13-31)$ to $8.5(1-11, \mathrm{p}=0.0078)$.

All medication was stopped prior to 24 -hour $\mathrm{pH}$ testing postPOEM; 13 patients (39\%) had completed 24-hour $\mathrm{pH}$ testing at the time of analysis. Two cases of acid reflux (positive 24-hour $\mathrm{pH}$, De-Meester scores of 24.7 and 14.8 , \% acid exposure time 5.3 and 6.2 minutes, respectively) were identified and managed with maintenance proton pump inhibitor.
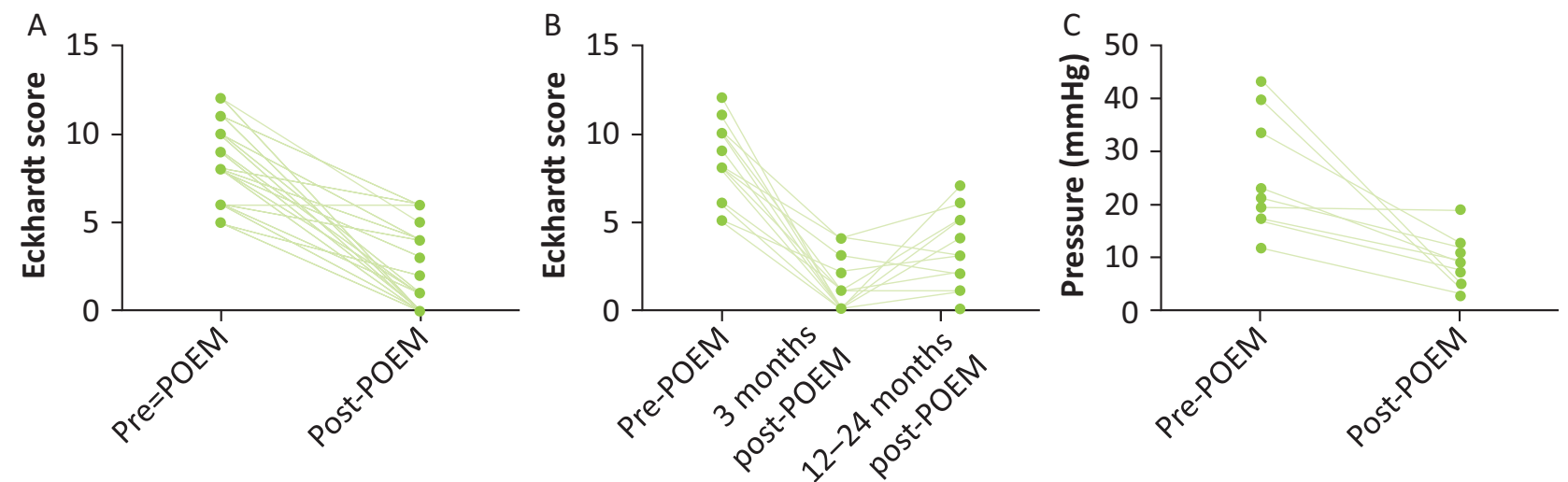

Fig 4. A - pre- and 3 month post-POEM Eckardt score ( $n=32)$; B - pre- and post-POEM Eckardt score at 3 and 12-24 months ( $n=16)$; C - pre- and post-POEM 4 -second integrated lower oesophageal pressure $(n=9)$. POEM = peroral endoscopic myotomy 


\section{Discussion}

POEM is a safe approach for primary achalasia that can be offered even to patients who have endured multiple previous endoscopic and surgical treatments. In our cohort, 58\% had received previous endoscopic or surgical treatment; higher than a worldwide cohort of 1,045 POEM procedures in which this figure was $38 \% .{ }^{23}$ Our patient cohort, therefore, represents a potentially more challenging patient population in whom a considerable success rate has been achieved.

All procedures were performed under general anaesthetic and in main operating theatres. An increased frequency of adverse events has been reported in patients in whom POEM was performed under intravenous sedation and air insufflation compared with endotracheal intubation and carbon dioxide insufflation. ${ }^{24}$ This is most likely attributable to general anaesthesia minimising unintentional patient movement and reducing the risk of mediastinal emphysema by achieving positive intrathoracic pressure as well as carbon dioxide being reabsorbed much faster than air, reinforcing our current practice. $^{24,25}$

Adverse events ranging from bleeding, perforation, subcutaneous emphysema, pneumoperitoneum, pneumothorax, pleural effusion and pneumonia have been reported after POEM. ${ }^{13,23}$ The majority of these resolve with conservative management, while perforation and bleeding can be managed endoscopically. Thoracotomy and drainage for persistent pleural effusion has been reported in 7/1,045 cases. ${ }^{23}$ There have been no mortalities and no intra-procedure conversion from POEM to surgery to date. In our cohort, there have been no peri-procedural complications to date. Two cases were abandoned because of the presence of oesophageal ulceration and intense mucosal fibrosis. Both were treated appropriately, discharged the same day and subsequently underwent POEM successfully in this cohort. A single patient had prolonged inpatient stay (12 days) because of unrelated medical issues (flare of gout and paroxysmal atrial fibrillation).

Of the patients in whom clinical success was not achieved at 3 months $(n=3)$ or sustained at $12-24$ months $(n=3), 66.6 \%$ received prior treatment for achalasia (patients 2, 3, 5 and 6, Table 2) with one receiving multiple treatments (patient 5: PD, BTX and LHM with Dor fundoplication). Patient 1 (Table 2) underwent POEM revision and has recovered well, with a current ES of 2; both procedures are included in this case series. Patient 2 subsequently reported improvement of ES to 3 at 6-month review and patients 3-6 are awaiting review with repeat manometry. Large scale studies are required to identify risk factors for POEM failure.

The incidence of GORD after POEM is variable in the collected literature to date and remains the most contentious factor in wider acceptance of the technique. Familiari et al ${ }^{26}$ identified that a lower IRP was associated with heartburn after POEM. Patient demographics, including age, body mass index, sigmoid oesophagus and previous therapies, did not significantly increase the risk of developing GORD. ${ }^{26}$ To date, there are no published data of patients routinely requiring anti-reflux procedures post-POEM. This is in contrast to LHM, which is routinely coupled with fundoplication. This may be due to the maintenance of extra-oesophageal anatomy at the gastro-oesophageal junction (GOJ), such as the phrenooesophageal ligament and preservation of the angle of this in
POEM. ${ }^{25}$ Larger prospective trials are required to evaluate risk factors for the development of GORD postoperatively.

It would be attractive to be able to assess success and predict the development of GORD using functional measurements. A multicentre retrospective analysis of peri-procedural EndoFLIP ${ }^{\circledR}$ measurements in 63 patients demonstrated a significantly greater final cross-sectional area and GOJ diameter in patients with clinically improved symptoms $(\mathrm{ES}<3)$, but also in patients who developed post-procedure pathological acid reflux. ${ }^{27}$ This study did not find a significant difference in the GOJ distensibility between responders versus non-responders, but is limited by heterogeneity in reporting, small sample size and relatively short follow-up period. ${ }^{27}$ Our EndoFlip results thus far appear to reflect this conclusion, but more data will be forthcoming. Other studies have demonstrated the utility of EndoFLIP ${ }^{\circledR}$ in assessing the success of PD or LHM, as well as in predicting treatment failure. ${ }^{28}$ Further study may yet establish a role for this technology in POEM.

Data thus far have shown that outcomes of LHM versus POEM are largely similar. A meta-analysis by Talukdar et al ${ }^{23}$ found that the time taken for POEM was significantly shorter than for LHM, but there was no significant difference in post-procedural pain, length of hospital stay or adverse events. There was no significant difference in the development of symptomatic reflux in this analysis, but $\mathrm{pH}$ studies were not performed. $^{23}$

Finally, in type III achalasia, significantly favourable outcomes for POEM versus LHM were demonstrated in terms of response to therapy and a shorter procedure time with fewer complications, despite longer myotomy. ${ }^{29}$ Given the utility of POEM in achieving a myotomy of greater length in the oesophagus, further studies may establish this as the treatment of choice in type III achalasia.

While the ability to achieve a longer myotomy in POEM compared with LHM is often cited as an advantage in general terms (regardless of subtype), the optimum length of myotomy is still a matter of debate. Although standard myotomy length is $10-15 \mathrm{~cm}$, a prospective study evaluating the short-term efficacy of a shorter myotomy (mean length $6.8 \mathrm{~cm}$ in 46 patients) demonstrated significant benefit up to 3 months. It may be that patients with predominant chest pain, or high-pressure oesophageal contractions, benefit from longer myotomy length (personal communication, Professor Inoue), but prospective study is required. ${ }^{24}$ In our case series, longer myotomy $(>12 \mathrm{~cm})$ was performed where chest pain was a predominant clinical feature and abnormal contractions evident at endoscopic examination.

\section{Conclusions}

While further observational studies, as well as comparator trials to other modalities, are warranted, POEM is now wellestablished internationally and considered a safe and effective treatment option for achalasia. The data from this, the first UK cohort of POEM for achalasia, demonstrate long-term success and safety rates in keeping with international reports in a potentially more challenging patient population.

\section{Author contributions}

All authors have significantly contributed to the data recruitment, analysis and manuscript preparation. 


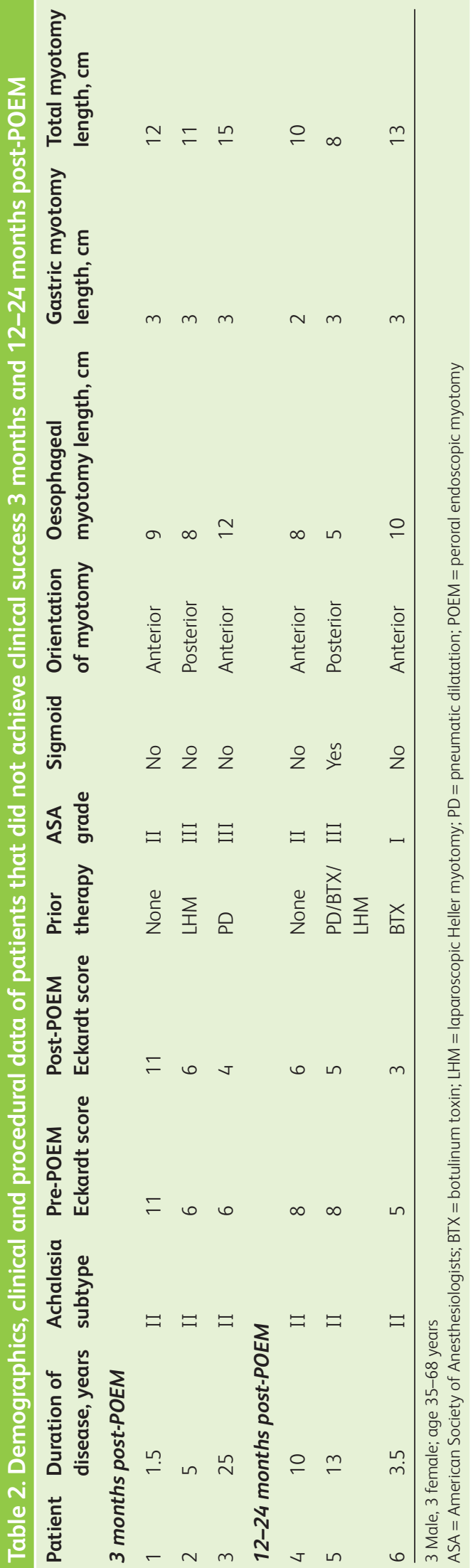




\section{Conflicts of interest}

The authors have no conflicts of interest to declare.

\section{References}

1 Mayberry JF. Epidemiology and demographics of achalasia. Gastrointest Endosc Clin N Am 2001;11:235-48.

2 Eckardt VF. Clinical presentations and complications of achalasia. Gastrointst Endosc Clin N Am 2001;11:281-92.

3 Pandolfino JE, Kwiatek MA, Nealis T et al. Achalasia: a new clinically relevant classification by high-resolution manometry. Gastroenterology 2008;135:1526-33.

4 Annese V, Bassotti G. Non-surgical treatment for esophageal achalasia. World J Gastroenterol 2006;12:5763-6.

5 Jung $\mathrm{H}$, Lee J, Lee $\mathrm{T}$ et al. Long-term outcomes of balloon dilatation versus botulinum toxin injection in patients with primary achalasia. Korean J Intern Med 2014;29:738-45.

6 Allaix ME, Patti MG. What is the best primary therapy for achalasia: medical or surgical treatment? Who owns achalasia? J Gastrointerest Surg 2013;17:1547-9.

7 Heller E. Extramukose Kardiaplastik beim chronischen kardiospasmus mit dilatation des oesophagus. Mitt Grenzgeb Med Chir 1914;27:141-9.

8 Boeckxstaens G, Zaninotto G, Richter JE. Achalasia. Lancet. 2014;383:83-93.

9 Shimi S, Nathanson LK, Cuschieri A. Laparoscopic cardiomyotomy for achalasia. J R Coll Surg Edinb 1991;36:152-4.

10 Ortega JA, Madureri V, Perez L. Endoscopic myotomy in the treatment of achalasia. Gastrointest Endosc 1980;26:8-10.

11 Pasricha PJ, Hawari R, Ahmed I et al. Submucosal endoscopic esophageal myotomy: a novel experimental approach for the treatment of achalasia. Endoscopy 2007;39:761-4.

12 Inoue $\mathrm{H}$, Minami $\mathrm{H}$, Kobayashi $\mathrm{Y}$ et al. Peroral endoscopic myotomy (POEM) for esophageal achalasia. Endoscopy 2010;42:265-71.

13 NOSCAR POEM White Paper Committee, Stavropoulos SN, Desilets DJ et al. Per-oral endoscopic myotomy white paper summary. Gastrointest Endosc 2014;80:1-15.

14 Maselli R, Inoue H, Misawa M et al. Peroral endoscopic myotomy (POEM) in a 3-year-old girl with severe growth retardation, achalasia, and Down syndrome. Endoscopy 2012;44(Suppl 2):285-7.

15 Stavropoulos S, Friedel D, Modayil R et al. Endoscopic approaches to treatment of achalasia. Therap Adv Gastroenterol 2013;6:115-35.

16 Inoue $\mathrm{H}$, Sato $\mathrm{H}$, Ikeda $\mathrm{H}$ et al. Per-oral endoscopic myotomy: a series of 500 patients. J Am Coll Surg 2015;221:256-64.

17 Tamhankar AP, Almogy G, Arian MA et al. Surgical management of hypertensive lower esophageal sphincter with dysphagia or chest pain. J Gastrointest Surg 2003;7:990-6.
18 Minami $\mathrm{H}$, Isomoto $\mathrm{H}$, Yamaguchi $\mathrm{N}$ et al. Peroral endoscopic myotomy (POEM) for diffuse esophageal spasm. Endoscopy 2014;46:E79-E81.

19 Stavropoulos SN, Modayil RJ, Friedel D et al. The international per oral endoscopic myotomy survey (IPOEMS): a snapshot of the global POEM experience. Surg Endosc 2013;27:3322-38.

20 Baldaque-Silva F, Marques M, Vilas-Boas F et al. New transillumination auxiliary technique for peroral endoscopic myotomy. Gastrointest Endosc 2014;79:544-5.

21 Familiari P, Gigante G, Marchese $M$ et al. EndoFLIP system for the intraoperative evaluation of peroral endoscopic myotomy. United European Gastroenterol J 2014;2:77-83.

22 Velanovich V, Vallance SR, Gusz JR et al. Quality of life scale for gastro-oesophageal reflux disease. J Am Coll Surg 1996;183:217-24.

23 Talukdar R, Inoue H, Reddy N. Efficacy of peroral endoscopic myotomy (POEM) in the treatment of achalasia: a systematic review and meta-analysis. Surgical Endosc 2015;29:3030-46.

24 Wang J, Tan N, Xiao Y et al. Safety and Efficacy of the modified peroral endoscopic myotomy with shorter myotomy for achalasia patients: a prospective study. Dis Esophagus 2015;28:720-7.

25 Bechara R, Ikeda H, Inoue H. Peroral endoscopic myotomy: an evolving treatment for achalasia. Nat Rev Gastroenterol Hepatol 2015;12:410-26.

26 Familiari P, Greco S, Gigante G et al. Gastroeoesophageal reflux disease after peroral endoscopic myotomy: analysis of clinical, procedural and functional factors associated with gastroeosophageal reflux disease and esophagitis. Dig Endosc 2016;28:33-41.

27 Ngamruengphong S, von Rahden BH, Filser J et al. Intraoperative measurement of esophagogastric junction cross-sectional area by impedance planimetry correlates with clinical outcomes of peroral endoscopic myotomy for achalasia: a multicenter study. Surg Endosc 2016;30:2886-94.

28 Rohof WO, Hirsch DP, Kessing BF et al. Efficacy of treatment for patients with achalasia depends on the distensibility of the esophagogastric junction. Gastroenterology 2012;143:328-35.

29 Khumbhari V, Tieu AH, Onimaru M et al. Peroral endoscopic myotomy (POEM) vs laparoscopic Heller myotomy (LHM) for the treatment of Type III achalasia in 75 patients: a multicenter comparative study. Endosc Int Open 2015;3:195-201.

Address for correspondence: Dr Shraddha Gulati, Department of Gastroenterology, Second Floor Hambledon Wing East, King's College Hospital, Denmark Hill, London SE5 9RS, UK. Email: shraddha.gulati@nhs.net 